\title{
Implante Valvular Aórtico Percutâneo (IVAP): Análise de uma Série de Casos Realizados com o Dispositivo Autoexpansível CoreValve ${ }^{\mathrm{TM}}$ Sem Uso de Pré-Dilatação
}

\author{
Guilherme L. M. Bernardi', Rogério Sarmento-Leite ${ }^{1}$, Paulo R. L. Prates ${ }^{1}$, Alexandre Schaan de Quadros ${ }^{1}$, \\ Imarilde Giusti', Tailur Grando', Paulo A. Salgado Filho'1, João Regis Lessa', Carlos A. M. Gottschall'1
}

\begin{abstract}
RESUMO
Introdução: $O$ implante valvular aórtico percutâneo (IVAP) vem se desenvolvendo rapidamente nos últimos anos. A manipulação da valva aórtica degenerada pode acarretar complicações. O IVAP direto, sem pré-dilatação com balão e com menor manipulação, seria uma alternativa. O objetivo deste estudo é apresentar uma série de 8 casos de IVAP direto com seguimento a médio prazo realizado no Instituto de Cardiologia do Rio Grande do Sul. Métodos: Série de 8 casos com descrição da técnica e resultados imediatos e a médio prazo do implante do dispositivo CoreValve ${ }^{\mathrm{TM}}$ sem valvuloplastia com balão. Resultados: No total, 7 pacientes do sexo masculino e 1 do sexo feminino, com média de idade de 76 anos e EuroSCORE logístico variando de $6 \%$ a $62 \%$, foram submetidos ao implante do dispositivo CoreValve ${ }^{\mathrm{TM}}$. Houve significativa queda dos gradientes entre o ventrículo esquerdo e a aorta. Foram registrados três casos de insucesso do implante, um óbito no período pós-implante imediato e um óbito aos 6 meses, sem relação com o procedimento. No seguimento de um ano, não houve novos casos de implante de marca-passo e eventos embólicos. Conclusões: O IVAP direto sem pré-dilatação com balão mostrou-se uma alternativa potencialmente eficaz. Quando realizado com sucesso, determina melhora dos sintomas, diminuição sustentável do gradiente transvalvar aórtico e aumento da área valvar aórtica. Não está claro, contudo, qual o paciente e qual a condição anatômica ideal para essa abordagem. Estudos adicionais e seguimento mais prolongado ainda são necessários para definir o exato papel e as precisas indicações dessa variação da técnica.
\end{abstract}

DESCRITORES: Valva aórtica. Estenose da valva aórtica. Próteses valvulares cardíacas. Implante de prótese de valva cardíaca. Dilatação com balão.

\section{ABSTRACT}

Percutaneous Aortic Valve Implantation (PAVI): Analysis of a Series of Cases Using the CoreValve $^{\mathrm{TM}}$ Self-Expandable Device Without Pre-Dilation

Background: Percutaneous aortic valve implantation (PAVI) has been developing rapidly in recent years. The manipulation of the degenerated aortic valve may lead to complications. PAVI without balloon pre-dilatation may be an alternative. The objective of this study is to report a series of 8 cases of direct PAVI with midterm follow-up, performed at the Instituto de Cardiologia do Rio Grande do Sul. Methods: Series of 8 cases with technical description and immediate and mid-term results of CoreValve ${ }^{T M}$ device implantation without balloon pre-dilatation. Results: A total of 7 male patients and 1 female patient, with mean age of 76 years and logistic EuroSCORE ranging from $6 \%$ to $62 \%$, were submitted to the CoreValve ${ }^{T M}$ device implantation. There was a significant decrease in the gradient between the left ventricle and the aorta. Three cases of implant failure, one death in the immediate post-implant period and one death at 6 months, with no relationship with the procedure, were reported. In the one-year follow-up, there were no new cases of pacemaker implantation and embolic events. Conclusions: Direct PAVI without balloon pre-dilatation proved to be a potentially effective alternative technique. When performed successfully, it improves symptoms, provides a sustainable aortic transvalvular gradient decrease and aortic valve area increase. It is not clear, however, which patients and what anatomical conditions are optimal for this approach. Additional studies and longer follow-up are still required to define the exact role and appropriate indications for this change in technique.

KEY-WORDS: Aortic valve. Aortic valve stenosis. Heart valve prosthesis. Heart valve prosthesis implantation. Balloon dilatation.

\footnotetext{
1 Instituto de Cardiologia do Rio Grande do Sul - Fundação Universitária de Cardiologia (IC-FUC) - Porto Alegre, RS, Brasil. Correspondência: Rogério Sarmento-Leite. Av. Princesa Isabel, 370 Santana - Porto Alegre, RS, Brasil - CEP 90620-000

E-mail: sarmentoleite@terra.com.br

Recebido em: 2/10/2011 • Aceito em: 6/12/2011
} 
0 número de procedimentos de implante valvular aórtico percutâneo (IVAP) vem crescendo de forma muito rápida no mundo todo. Hoje, já existem evidências científicas robustas de que é um método seguro e efetivo para pacientes com estenose aórtica grave sintomática e considerados inoperáveis ou de alto risco cirúrgico. ${ }^{1-3}$ Essa tecnologia vem se desenvolvendo extraordinariamente durante os últimos anos com novas gerações de dispositivos, redução dos perfis, novas rotas de acesso e aprimoramento da técnica, permitindo maior segurança e eficácia do procedimento. ${ }^{4,5}$

As taxas de sucesso do procedimento são altas e as complicações relacionadas especificamente ao método são relativamente baixas, se avaliado o perfil de risco dos pacientes submetidos ao procedimento. ${ }^{6,7}$ Os distúrbios de condução atrioventricular necessitando de implante de marca-passo permanente, as complicações relacionadas ao acesso vascular e os fenômenos embólicos são as principais complicações do procedimento. ${ }^{8}$ As complicações mais conhecidas são principalmente os fenômenos embólicos causados pela liberação de fragmentos em decorrência da manipulação de uma válvula aórtica degenerada e calcificada e os distúrbios de condução pela proximidade do ânulo aórtico e da via de saída do ventrículo esquerdo com o sistema de condução. Essas complicações, relatadas em todos os registros e ensaios clínicos, são relacionadas à manipulação da valva nativa e às diversas etapas do procedimento.

Desde o início da utilização do método com a prótese autoexpansível CoreValve ${ }^{\mathrm{TM}}$ (CoreValve Inc., Irvine, Estados Unidos), a preparação da valva nativa com valvuloplastia com balão antes da liberação da prótese era obrigatória, com o objetivo de facilitar a liberação e a expansão adequada da prótese, reduzindo a contraforça radial oferecida pela valva nativa degenerada. Contudo, a valvuloplastia com balão pode potencializar os riscos de embolização distal e de acidente vascular cerebral, distúrbios de condução e regurgitação aórtica grave com instabilidade hemodinâmica. Um estudo recentemente publicado sugere que essa pré-dilatação pode ser suprimida sem prejuízos. ${ }^{9} \mathrm{~A}$ presente série retrata a experiência da instituição.

\section{MÉTODOS}

\section{Desenho do estudo e critérios de seleção}

Série de 8 casos de IVAP em pacientes com estenose aórtica grave e com alto risco cirúrgico submetidos ao procedimento com implante direto da prótese CoreVal$v^{\mathrm{TM}}$ sem pré-dilatação. Todos os casos foram realizados no Instituto de Cardiologia do Rio Grande do Sul e representam 8 dos 27 casos já realizados na instituição desde outubro de 2008.

Foram selecionados pacientes com estenose aórtica grave (área valvar $<1 \mathrm{~cm}^{2}$ ), considerados de alto risco cirúrgico e com repercussão clínica, como sintomas de dor torácica ou dispneia limitante, tonturas, pré-síncope ou síncope, arritmia ventricular ou disfunção de bioprótese aórtica.

Todos os casos foram avaliados por uma equipe multidisciplinar composta por um cardiologista clínico, um cardiologista intervencionista e um cirurgião cardíaco, sendo a troca valvular aórtica por cirurgia considerada com maior potencial risco de mortalidade perioperatória e de possíveis complicações pós-operatórias.

A triagem dos pacientes envolveu a avaliação de condições anatômicas e funcionais favoráveis para o implante da prótese CoreValve ${ }^{\mathrm{TM}}$. Para tanto, foram realizados ecocardiograma transtorácico com Doppler em cores, cineangiocoronariografia e angiotomografia de múltiplos detectores, conforme o caso. Os dados clínicos e angiográficos foram incluídos em formulários de acordo com o protocolo de avaliação padrão do fabricante, e a análise dessas características determinou a inclusão dos pacientes no estudo. Os pacientes elegíveis eram orientados sobre os riscos e benefícios do procedimento e todos assinaram o termo de consentimento livre informado.

\section{Descrição do dispositivo e do procedimento}

Foi utilizada a terceira geração da prótese valvular aórtica CoreValve ${ }^{\mathrm{TM}}$, que consiste de uma bioprótese de três folhetos de pericárdio porcino montados e suturados e um stent autoexpansível de nitinol. As características da prótese e a técnica padrão de implante foram previamente descritas. ${ }^{10} \mathrm{Na}$ presente série, todos os procedimentos foram realizados sem pré-dilatação. A opção por não pré-dilatar foi baseada em critérios subjetivos, que consideravam, sobretudo, o grau de calcificação, o diâmetro do anel e o risco de embolização de debris.

Todos os pacientes receberam $200 \mathrm{mg}$ de ácido acetilsalićlico e $300 \mathrm{mg}$ de clopidogrel na noite anterior. Os procedimentos foram realizados sob anestesia geral e em vigência de anticoagulação com heparina não-fracionada ajustada pelo peso para se atingir tempo de coagulação ativada de 300 segundos a 350 segundos. Um eletrodo de marca-passo cardíaco temporário era introduzido já no início do procedimento pela veia jugular direita, para prevenção de bradiarritmias. Não foi necessário aumentar a frequência cardíaca (rapid pacing) como é feito nos procedimentos com valvuloplastia para evitar migração do balão. O acesso vascular arterial principal para entrega da prótese era obtido com dissecção da artéria femoral comum ou ilíaca, com o objetivo de se canular um vaso com pelo menos $6 \mathrm{~mm}$ de diâmetro, permitindo a introdução de uma bainha $18 \mathrm{~F}$. Outro acesso arterial no membro inferior contralateral era necessário para posicionamento de um cateter pigtail $5 \mathrm{~F}$, utilizado para injeção de contraste e auxílio no momento do posicionamento e 
da liberação da prótese. O ventrículo esquerdo era acessado retrogradamente com um fio-guia hidrofílico de troca introduzido através de um cateter AL1 $5 \mathrm{~F}$ ou 6 F. Posteriormente, esse cateter era substituído por um pigtail, medindo-se o gradiente transvalvar e mantendo-se o fio-guia Amplatz super-stiff na cavidade. Em seguida, era liberada a prótese CoreValve ${ }^{T M}$, por meio de implante direto, com tamanho pré-definido de acordo com o tamanho do anel valvar.

Parâmetros clínicos e hemodinâmicos eram continuamente avaliados durante o procedimento. Aortografias contrastadas foram realizadas antes, durante e após o implante da válvula para auxiliar o posicionamento e a liberação da prótese e avaliar vazamentos ou regurgitação aórtica residual. Ecocardiografia transesofágica foi utilizada de forma rotineira. Após o término dos procedimentos, os pacientes eram encaminhados para uma unidade de tratamento intensivo, onde permaneciam por pelo menos 48 horas para observação. O marca-passo temporário era mantido por todo esse período. Ecocardiograma transtorácico foi realizado antes da alta, com a recomendação para os pacientes de manter ácido acetilsalicílico (100 mg/dia) indefinidamente e clopidogrel $(75 \mathrm{mg} / \mathrm{dia})$ por pelo menos 6 meses. Todos os pacientes foram reavaliados com consulta clínica, eletrocardiograma e ecocardiograma no seguimento de 1 mês, 6 meses e 1 ano.

\section{RESULTADOS}

A Tabela 1 apresenta as características de base, com dados demográficos, fatores de risco e EuroSCORE logístico dos 8 pacientes submetidos ao IVAP direto, dos quais 7 eram do sexo masculino e 1 do sexo feminino, com média de idade de 76 anos e EuroSCORE logístico médio de $21 \%$, variando de $6 \%$ a $62 \%$. A etiologia era degenerativa em todos os casos, e em um caso o paciente apresentava degeneração de prótese aórtica biológica e dupla lesão aórtica com predomínio de regurgitação aórtica grave.

O acesso arterial para introdução da prótese CoreValve ${ }^{\mathrm{TM}}$ foi realizado por dissecção da artéria femoral nos 8 pacientes, possibilitando o uso de bainha de 18 F. Por questões anatômicas e de rotina, a primeira escolha foi a artéria femoral direita. Todos os procedimentos foram realizados com anestesia geral.

O ecocardiograma transesofágico no periprocedimento foi realizado em todos os pacientes, mostrando-se altamente útil na seleção do tamanho e no posicionamento adequado da prótese, na avaliação da função cardíaca durante o procedimento, e na pronta avaliação do funcionamento do implante valvular logo após ser liberado.

\section{Resultados clínicos e laboratoriais de seguimento}

Houve sucesso do implante em 5 pacientes, de acordo com os critérios do Valvar Academic Research
TABELA 1

Características de base dos pacientes

\begin{tabular}{|c|c|}
\hline & $\mathbf{n}=\mathbf{8}$ \\
\hline \multicolumn{2}{|l|}{ Sexo, $\mathrm{n}$} \\
\hline Masculino & 7 \\
\hline Feminino & 1 \\
\hline Média de idade, anos & $76 \pm 11,3$ \\
\hline EuroSCORE logístico médio, \% & $21 \pm 20$ \\
\hline \multicolumn{2}{|l|}{ Principais comorbidades, n (\%) } \\
\hline Hipertensão arterial sistêmica & $5(62,5)$ \\
\hline Diabetes melito & $4(50)$ \\
\hline Doença arterial coronária & $7(87,5)$ \\
\hline Infarto agudo do miocárdio prévio & $5(62,5)$ \\
\hline Intervenção coronária percutânea prévia & $4(50)$ \\
\hline $\begin{array}{l}\text { Cirurgia de revascularização } \\
\text { do miocárdio prévia }\end{array}$ & $3(37,5)$ \\
\hline Troca valvar aórtica prévia & $1(12,5)$ \\
\hline Doença vascular periférica & $2(25)$ \\
\hline $\begin{array}{l}\text { Acidente vascular cerebral ou } \\
\text { ataque isquêmico transitório prévio }\end{array}$ & $3(37,5)$ \\
\hline Hipertensão pulmonar & $1(12,5)$ \\
\hline Insuficiência renal (DCE < 60 ml/min) & $7(87,5)$ \\
\hline Doença pulmonar obstrutiva crônica & $1(12,5)$ \\
\hline Marca-passo prévio & $4(50)$ \\
\hline Classe funcional (NYHA) I & 0 \\
\hline Classe funcional (NYHA) II & $2(25)$ \\
\hline Classe funcional (NYHA) III & $5(62,5)$ \\
\hline Classe funcional (NYHA) IV & $1(12,5)$ \\
\hline
\end{tabular}

Idade e EuroSCORE logístico apresentados como média \pm desvio padrão.

DCE = depuração da creatinina endógena; $\mathrm{n}=$ número de pacientes; NYHA $=$ New York Heart Association.

Consortium (VARC) ${ }^{11}$. Nesses pacientes, o procedimento foi tecnicamente simples, sem intercorrências. A prótese cruzou facilmente a valva aórtica, penetrando na cavidade ventricular esquerda. A liberação transcorreu da forma habitual, com redução imediata dos gradientes entre o ventrículo esquerdo e a aorta e ausência de regurgitação aórtica ecocardiográfica e angiograficamente expressiva. Entretanto, em 3 casos observamos insucesso, mais precisamente nos pacientes 2, 7 e 8 . A Tabela 2 apresenta as características individuais clínicas, ecocardiográficas e de seguimento. No primeiro caso (paciente 2), o dispositivo embolizou para a aorta ascendente durante sua liberação, ficando acima do plano valvar. Tentou-se, então, recolher o dispositivo para retirá-lo por meio do introdutor. Todavia, durante a tração não foi possível encapá-lo novamente, sendo necessária sua fixação na aorta descendente. Na sequência, foi implantada uma segunda prótese CoreVal$\mathrm{ve}^{\mathrm{TM}}$, que ficou posicionada de forma adequada. A Figura 1 demonstra o achado radiológico, permitindo 
TABELA 2

Características individuais clínicas, ecocardiográficas e de seguimento

\begin{tabular}{|c|c|c|c|c|c|c|c|c|c|}
\hline & $\begin{array}{c}\text { Idade } \\
\text { em anos } \\
\text { (sexo) }\end{array}$ & $\begin{array}{c}\text { Classe } \\
\text { funcional } \\
(\mathrm{NYHA})\end{array}$ & $\begin{array}{c}\text { EuroSCORE } \\
\log (\%)\end{array}$ & $\begin{array}{c}\text { Área } \\
\text { valvar } \\
\text { aórtica } \\
\left(\mathrm{cm}^{2}\right)\end{array}$ & $\begin{array}{c}\text { Diâmetro } \\
\text { do anel } \\
\text { aórtico } \\
(\mathrm{mm})\end{array}$ & $\begin{array}{l}\text { Gradiente } \\
\text { médio } \\
\text { VE-Ao pré } \\
(\mathrm{mmHg})\end{array}$ & $\begin{array}{l}\text { Gradiente } \\
\text { médio } \\
\text { VE-Ao pós } \\
\text { (mmHg) }\end{array}$ & $\begin{array}{c}\text { Sucesso } \\
\text { do } \\
\text { implante* }\end{array}$ & Seguimento \\
\hline Paciente 1 & $82(\mathrm{M})$ & III & 17,2 & 0,6 & 23 & 40 & 10 & Sim & $\begin{array}{l}\text { Óbito } 6 \text { meses após } \\
\text { por mieloma múltiplo }\end{array}$ \\
\hline Paciente $2^{+}$ & $87(M)$ & III & 46 & ND & 22 & 15 & 11 & Não & Estável após 1 ano \\
\hline Paciente 3 & $83(\mathrm{~F})$ & $\mathrm{N}$ & 13,8 & 0,6 & 22 & 35 & 6 & Sim & Estável após 1 ano \\
\hline Paciente 4 & $89(\mathrm{M})$ & III & 62,9 & 0,8 & 27 & 44 & 5 & Sim & Estável após 1 ano \\
\hline Paciente 5 & $62(M)$ & III & 8,3 & 0,6 & 24 & 32 & 7 & Sim & Estável após 1 ano \\
\hline Paciente 6 & $64(M)$ & III & 10,9 & 0,6 & 23 & 41 & 7 & Sim & Estável após 1 ano \\
\hline Paciente 7 & $64(M)$ & $\|$ & 8,1 & 0,6 & 22 & 52 & 8 & Não & $\begin{array}{c}\text { Óbito no } \\
\text { pós-implante imediato }\end{array}$ \\
\hline Paciente 8 & $83(\mathrm{M})$ & $\|$ & 6,6 & 0,6 & 24 & 76 & 7 & Não & Estável após 6 meses \\
\hline
\end{tabular}

a visualização das duas próteses. O paciente teve excelente evolução clínica e encontra-se clinicamente bem, com significativa redução dos sintomas e com melhora sustentada da qualidade de vida. No segundo caso de insucesso (paciente 7), houve grande dificuldade de posicionamento inicial da prótese, com duas migrações no momento da liberação. Na primeira, foi possível reencapar o dispositivo e utilizá-lo novamente; na segunda oportunidade, porém, após a soltura do último anel, a prótese literalmente saltou para fora do ventrículo e ficou alojada na porção supra-anelar. Como não foi constatado comprometimento dos óstios coronários, optou-se pelo implante de uma segunda CoreValve $^{\mathrm{TM}}$ (Figura 2). Somando-se a isso, em decorrência da grave doença vascular periférica, houve laceração da artéria femoral direita, que necessitou de reparo cirúrgico, mas determinou perda sanguínea significativa, com necessidade de politransfusão e prolongado tempo de anestesia geral. A despeito de a segunda CoreValve ${ }^{\mathrm{TM}}$ ter ficado adequadamente implantada e normofuncionante, o paciente evoluiu com quadro de choque cardiogênico e faleceu algumas horas após o procedimento. No terceiro caso de insucesso (paciente 8), houve má expansão da estrutura de nitinol pelo alto grau de calcificação da válvula aórtica nativa, culminando com regurgitação aórtica grave não solucionada com pós-dilatação com balão. Esse paciente evoluiu com insuficiência cardíaca progressiva e 7 dias após o IVAP foi submetido a cirurgia, com explante da CoreValve ${ }^{\mathrm{TM}}$ e troca cirúrgica da válvula aórtica com prótese biológica, evoluindo com sucesso.

No seguimento tardio dessa série, um dos pacientes (paciente 1) faleceu 6 meses após o IVAP por complicações de um mieloma múltiplo, mas a prótese

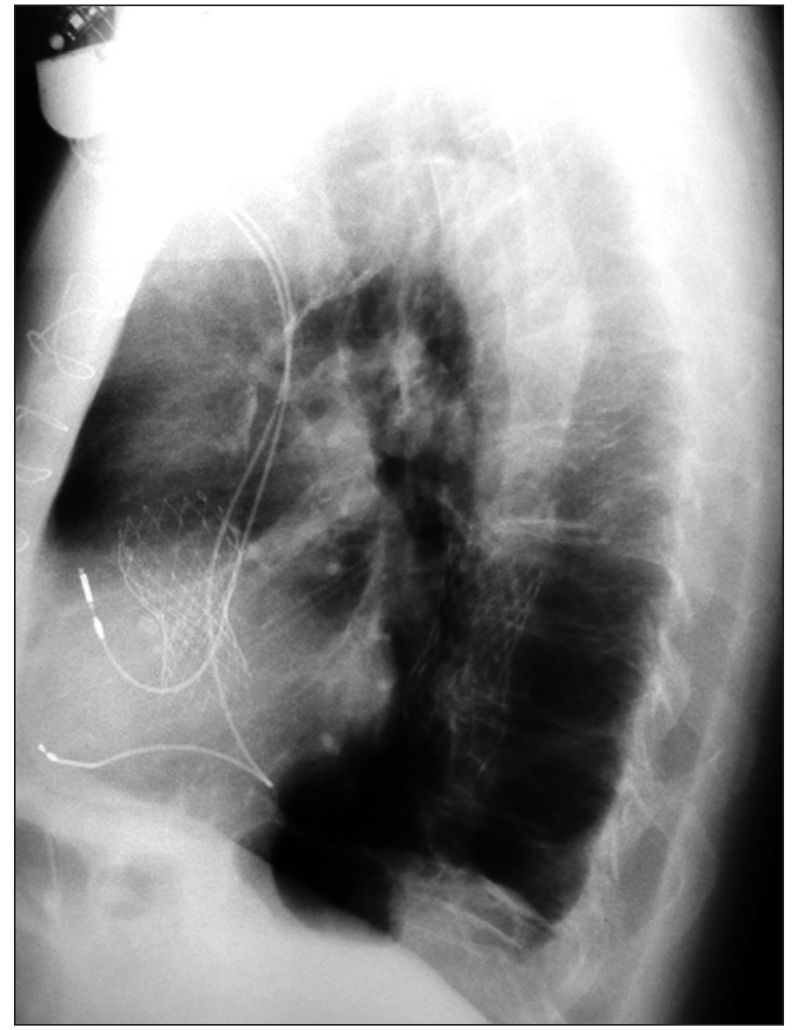

Figura 1 - Radiografia de tórax em perfil esquerdo.

estava funcionando adequadamente. Os demais pacientes apresentaram significativa mudança favorável da qualidade de vida, demonstrada pela melhora de sua classe funcional (CF) e encontram-se estáveis, com 


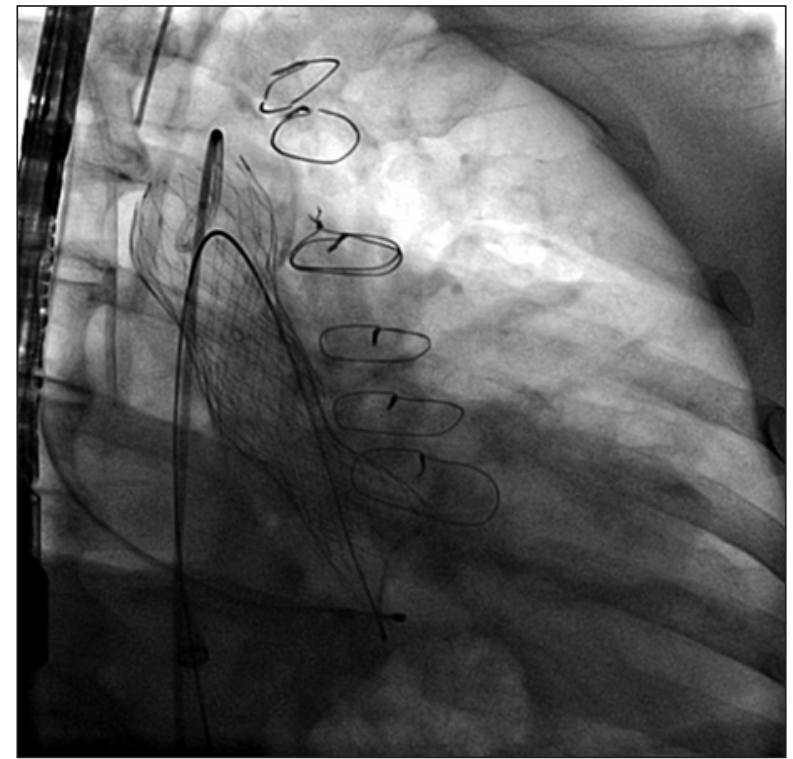

Figura 2 - Duas próteses CoreValve ${ }^{\mathrm{TM}}$ em overlapping (valve-in-valve).

CF I ou II de acordo com a classificação da New York Heart Association (NYHA).

Dos 8 pacientes avaliados, 4 já vinham em uso de marca-passo definitivo, um dos quais com marca-passo associado a cardiodesfibrilador. No controle eletrocardiográfico imediato e no seguimento, não foi observado distúrbio de condução atrioventricular ou intraventricular nos 4 pacientes que não apresentavam implante prévio de marca-passo. Não ocorreram eventos embólicos, principalmente isquêmicos cerebrais, em um ano.

A avaliação ecocardiográfica no seguimento demonstrou queda sustentável dos gradientes ventrículo esquerdo-aorta e manutenção do aumento da área valvar aórtica.

\section{DISCUSSÃO}

No presente estudo, são descritos os dados clínicos relacionados ao procedimento e ao seguimento dos 8 pacientes submetido a IVAP com a prótese CoreValve ${ }^{\mathrm{TM}}$ sem valvuloplastia aórtica com balão no Instituto de Cardiologia do Rio Grande do Sul. O implante direto foi realizado com sucesso na maioria dos pacientes, ocorrendo 3 casos de insucesso. Foram registrados 2 óbitos, um dos quais não relacionado ao procedimento. Não ocorreram complicações embólicas ou novos implantes de marca-passo no seguimento de um ano.

Desde o relato do primeiro IVAP, a valvuloplastia como pré-dilatação tem sido considerada obrigatória e parte integrante do procedimento. A justificativa teórica, mas lógica e plausível, seria preparar a válvula para o implante da prótese. Poucas são as evidências para o IVAP, principalmente com a prótese autoex- pansível CoreValve ${ }^{T M}$ sem uso prévio de valvuloplastia com balão. Um estudo piloto de caso controle multicêntrico com 186 pacientes comparou as duas técnicas, com e sem pré-dilatação, com a prótese CoraValve ${ }^{\mathrm{TM}}$ e observou resultados similares com as duas técnicas, demonstrando ser potencialmente viável o implante direto como estratégia para facilitar o procedimento e aumentar sua segurança. ${ }^{9}$ Certamente isso retrata o atual desenvolvimento tecnológico do dispositivo, que tem baixo perfil, boa navegabilidade e um sistema de liberação que permite mais precisão. Além disso, ressalta os grandes atrativos do implante direto, que são o menor tempo de procedimento, a menor manipulação da valva nativa degenerada, o que poderia teoricamente diminuir as complicações embólicas e os distúrbios de condução, e a não utilização do rapid-pacing, que, em pacientes com baixa fração de ejeção, poderia levar à instabilidade hemodinâmica. Todavia, esses fatos ainda não foram demonstrados por estudos de maior peso e sua reprodutibilidade também foi muito pouco descrita na prática clínica.

O que se questiona a respeito da não pré-dilatação é que não haveria um "preparo" adequado da valva nativa para receber a prótese autoexpansível, culminando em defeitos de expansão e expansão não-circular da prótese e causando persistências do gradiente ventrículo esquerdo-aorta. A grande força radial do dispositivo talvez seja suficiente para evitar ou minimizar esses efeitos. O problema é como selecionar o bom ou o mau candidato para tal. Não existem diretrizes, normas ou recomendações específicas para esse fim, e o grau de calcificação parece ser o que mais pesa na decisão. O paradoxo desse conceito é que ele pode ser compreendido por duas óticas. Uma grande carga de cálcio tem, em tese, mais chance de desprender uma placa e provocar embolização quando rompida pelo balão durante a valvuloplastia, razão para defendermos o implante direto. No entanto, se pensarmos que uma elevada carga de cálcio pode prejudicar a adequada expansão da válvula, pode-se pregar que a valvuloplastia está recomendada. Assim, existem argumentos para os dois lados e uma necessidade de evidências mais robustas para se firmar uma conclusão definitiva.

Nossa série é limitada pelo pequeno número de pacientes e casos muito selecionados sem grupo controle, mas todos aqueles que receberam alta após o procedimento apresentaram queda esperada do gradiente ventrículo esquerdo-aorta e aumento da área valvar aórtica sustentada similares ao observado nos casos realizados com pré-dilatação. Os casos de insucesso da abordagem direta podem ter sido secundários a questões pontuais, como ser um procedimento de "válvula em válvula", em que o referencial anatômico e o atrito do tecido anular degenerado que permite segurar a CoreValve ${ }^{\mathrm{TM}}$ estão ausentes ou pela maciça calcificação presente em outro, mas os resultados não permitem segurança absoluta para adoção 
dessa técnica como padrão. Isso reforça que todos os casos devem ser individualizados e a abordagem e o tipo de procedimento para cada paciente podem ser influenciados por fatores clínicos e anatômicos. Estudos maiores e desenhados para esse fim devem ser realizados para dirimir essas dúvidas.

\section{CONCLUSÕES}

O IVAP direto sem pré-dilatação com balão mostrou-se uma alternativa potencialmente eficaz. Quando realizado com sucesso, determina melhora dos sintomas, diminuição sustentável do gradiente transvalvar aórtico e aumento da área valvar aórtica. Não está claro, contudo, qual o paciente e qual a condição anatômica ideal para essa abordagem.

\section{CONFLITO DE INTERESSES}

Rogério Sarmento-Leite é palestrante em eventos ou atividades patrocinadas pela Medtronic. Os demais autores declaram não haver conflito de interesses relacionado a este manuscrito.

\section{REFERÊNCIAS}

1. Leon MB, Smith CR, Mack M, Miller DC, Moses JW, Svensson LG, et al. Transcatheter aortic-valve implantation for aortic stenosis in patients who cannot undergo surgery. $N$ Engl J Med. 2010;363(17):1597-607.

2. Smith CR, Leon MB, Mack MJ, Miller DC, Moses JW, Svensson LG, et al. Transcatheter versus surgical aortic-valve replacement in high-risk patients. N Engl J Med. 2011;364(23): 2187-98.

3. Tarasoutchi F, Montera MW, Grinberg M, Barbosa MR, Piñeiro DJ, Sánchez CRM, et al. Diretriz Brasileira de Valvopatias/
I Diretriz Interamericana de Valvopatias - SIAC 2011. Arq Bras Cardiol. 2011;97(5 Supl. 3):1-67.

4. Petronio AS, De Carlo M, Bedogni F, Marzocchi A, Klugmann $S$, Maisano F, et al. Safety and efficacy of the subclavian approach for transcatheter aortic valve implantation with the CoreValve Revalving system. Cir Cardiovasc Interv. 2010;3(4): 359-66.

5. Al-Lamee R, Godino C, Colombo A. Transcatheter aortic valve implantation: current principles of patient and technique selection and future perspectives. Cir Cardiovasc Interv. 2011;4(4):387-95.

6. Grube E, Schuler G, Buellesfeld L, Gerckens U, Linke A, Wenaweser $\mathrm{P}$, et al. Percutaneous aortic valve replacement for severe aortic stenosis in high-risk patients using the second- and current third-generation self-expanding CoreValve prosthesis: device success and 30-day clinical outcome. J Am Coll Cardiol. 2007;50(1):69-76.

7. Grube E, Buellesfeld L, Mueller R, Sauren B, Zickmann B, Nair D, et al. Progress and current status of percutaneous aortic valve replacement: results of three device generations of the CoreValve Revalving system. Cir Cardiovasc Interv. 2008;1(3):167-75.

8. Sarmento-Leite R, Quadros AS, Prates PRL, Voltolini I, Conti E, Giusti I, et al. Marca-passo permanente após implante percutâneo valvular aórtico: a necessidade é maior que imaginávamos? Rev Bras Cardiol Invasiva. 2009;17(4):476-83.

9. Grube E, Naber C, Abizaid A, Sousa E, Mendiz O, Lemos $P$, et al. Feasibility of transcatheter aortic valve implantation without balloon pre-dilation: a pilot study. JACC CardiovasC Interv. 2011;4(7):751-7.

10. Sarmento-Leite R, Quadros AS, Prates PRL, Zanatta LG, Salgado Filho PA, Grando T, et al. Implante valvular aórtico percutâneo: experiência inicial do Sul do Brasil. Rev Bras Cardiol Invasiva. 2008;16(4):398-405.

11. Leon MB, Piazza N, Nikolsky E, Blackstone EH, Cutlip DE, Kappetein AP, et al. Standardized endpoint definitions for Transcatheter Aortic Valve Implantation clinical trials: a consensus report from the Valve Academic Research Consortium. J Am Coll Cardiol. 2011;57(3):253-69. 